15-1 | 2018

Mettre en scène l'activité : les nouvelles figures de la simulation

\title{
La simulation de l'activité en conception ergonomique : acquis et perspectives
}

Activity simulation in design: achievements and perspectives

\section{Laurent Van Belleghem}

\section{OpenEdition}

\section{Journals}

Édition électronique

URL : http://journals.openedition.org/activites/3129

DOI : 10.4000/activites.3129

ISSN : 1765-2723

Éditeur

ARPACT - Association Recherches et Pratiques sur les ACTivités

Référence électronique

Laurent Van Belleghem, «La simulation de l'activité en conception ergonomique

acquis et perspectives », Activités [En ligne], 15-1 | 2018, mis en ligne le 15 avril 2018, consulté le 19 avril 2019. URL : http://journals.openedition.org/activites/3129; DOI : 10.4000/activites.3129

Ce document a été généré automatiquement le 19 avril 2019

\section{c) $($ ) $(9)$}

Activités est mis à disposition selon les termes de la licence Creative Commons Attribution - Pas d'Utilisation Commerciale - Pas de Modification 4.0 International. 


\title{
La simulation de l'activité en conception ergonomique : acquis et perspectives
}

Activity simulation in design: achievements and perspectives

\author{
Laurent Van Belleghem
}

\section{NOTE DE L'ÉDITEUR}

Article soumis le 21/12/2017, accepté le 24/03/2018

\section{Introduction}

Le recours à la simulation de l'activité en conception de situations de travail fait l'objet d'un intérêt renouvelé tant en France (Heddad, 2016; Barcellini, Van Belleghem, \& Daniellou, 2013) qu'à l'international, notamment au Brésil (Braatz, Tonin, Fontes, \& Menegon, 2013 ; Braatz, \& Paravizo, 2017) et au Danemark (Andersen, \& Broberg, 2015, 2016). Trente ans après ses premières formalisations dans le champ de l'ergonomie de l'activité, ce regain d'intérêt ne marque pas tant une redécouverte qu'un approfondissement de ses principes théoriques et méthodologiques en même temps qu'un élargissement de ses perspectives. D'abord outil d'aide à la conduite de projet (Maline, 1994), la simulation de l'activité révèle en effet des potentialités d'application diversifiées et particulièrement puissantes pour soutenir les réflexions stratégiques sur le travail et ses transformations possibles. Largement éprouvée pour concevoir des artefacts ou définir les dimensions physiques ou spatiales des postes de travail, de nouveaux usages de la simulation émergent pour concevoir des organisations ou systèmes de management, ayant donné naissance à des méthodologies spécifiques de simulation organisationnelle (Van Belleghem, 2012; Daniellou, Legal, \& Promé, 2014; Mellon, 2015) ou managériale (Van Belleghem, \& Guerry, 2016; Besnard, \& Levy, 2017). Ce mouvement invite à 
considérer de multiples dimensions des situations de travail comme des objets de conception légitimes. La gouvernance des entreprises, le métier, le dialogue social, la confiance au travail, la coopération, la discussion sur le travail, etc. sont ainsi autant de champs structurants du travail que les entreprises ne savent généralement pas appréhender et qu'une méthodologie de simulation de l'activité pourrait aider à mieux approcher pour en concevoir les conditions d'avènement. Par ailleurs, la reconnaissance de processus développementaux à l'œuvre au cours des simulations (Barcellini, Van Belleghem, \& Daniellou, 2013) ouvre des champs d'application non uniquement orientés vers le projet, mais aussi vers les sujets, suivant l'ambition d'une ergonomie constructive (Falzon, 2013).

2 Ouvrir ces perspectives d'usage de la simulation pour les étendre à une vaste gamme de situations et de problématiques nécessite de mieux en définir les mécanismes. Trois enjeux doivent être tenus pour cela.

- Tout d'abord, si l'activité simulée doit rendre compte de l'activité réelle, elle doit recréer ses conditions d'exercice et de développement. Dès lors, l'élaboration d'un modèle de la simulation de l'activité ne peut se passer d'un modèle de l'activité en situation. C'est un enjeu théorique.

- Ensuite, la simulation de l'activité étant elle-même une activité, elle invite à penser et à construire la situation de simulation (Béguin, \& Weill-Fassina, 1997b) comme une situation d'activité de simulation de l'activité. Les caractéristiques du dispositif de simulation doivent pour cela être précisément définis, qu'il s'agisse des finalités à poursuivre, des acteurs à réunir, des supports de simulation à créer, des modalités d'animation à mettre en œuvre... pour soutenir l'activité de simulation de l'activité. C'est un enjeu méthodologique.

- Enfin, la simulation de l'activité étant mobilisée dans des projets de transformation du travail, elle doit contribuer concrètement à la définition des caractéristiques des futurs systèmes de prescription, mais aussi à leur appropriation par les travailleurs. C'est un enjeu opérationnel, visant dans un même mouvement la conception des situations et le développement de l'activité qui doit les investir.

3 Cet article se propose de mieux donner à voir le potentiel d'usage de la simulation de l'activité et ses perspectives d'application en conception ergonomique. Il est organisé en trois parties. La première rappelle certains acquis fondamentaux de la simulation dans l'histoire de l'ergonomie de l'activité, devant permettre de poser le socle sur lequel dresser ses développements à venir. La deuxième tente d'approfondir théoriquement les mécanismes de la simulation de l'activité en lien avec l'activité elle-même. La troisième explore les perspectives de la simulation, d'abord en exploitant ses facultés de rétroaction sur le système de prescription et sur les logiques d'action des sujets agissants, ensuite en élargissant les objets de conception qu'elle permet d'approcher.

\section{Les acquis de la simulation en ergonomie de l'activité}

\subsection{La simulation de l'activité : surmonter le paradoxe de l'ergonomie de conception}

Dans le courant de l'ergonomie francophone, l'idée du recours à la simulation de l'activité prend naissance au milieu des années 1980. L'objectif est alors de pallier les écueils 
constatés de projets conduits avec une trop faible référence au travail réel, générant des effets négatifs observables en termes de santé, de sécurité ou d'efficacité (Barcellini, Van Belleghem, \& Daniellou, 2013). L'enjeu se fait alors sentir d'une conception centrée sur l'activité de travail, révélant dans le même temps un paradoxe (Theureau, \& Pinsky, 1984) : dans un projet de conception, l'activité future de travail n'existant pas encore, on ne peut pas concevoir "autour». La simulation de l'activité est proposée comme un moyen de surmonter ce "paradoxe de l'ergonomie de conception", en invitant les travailleurs à anticiper « l'espace des formes possibles d'activité future » (Daniellou, 2004, p. 360) induit par les choix de conception élaborés par les concepteurs. Mises en œuvre dans le cadre de démarches participatives, les simulations visent à évaluer et faire évoluer les choix de conception réalisés dans le projet permettant la construction du meilleur compromis entre les exigences des participants (Daniellou, 1992 ; Maline, 1994). L'importance des interactions, voire des confrontations, socio-cognitives dans le cadre d'un processus de conception engageant une pluralité d'acteurs est particulièrement soulignée (Garrigou, 1992 ; Béguin, \& Weill-Fassina, 1997a). Ce positionnement marque une rupture dans la façon dont l'ergonomie envisage son apport à la transformation des situations de travail. Sa finalité vise moins, désormais, la proposition de recommandations issues d'un diagnostic initial que les concepteurs seraient chargés d'intégrer que "la mise en ceuvre d'une démarche de conduite de projet organisée par l'ergonome visant l'enrichissement des projets de conception par la prise en compte du travail » (Barcellini, 2015, p. 90). La simulation du travail, au cœur de cette démarche, fait alors l'objet d'un développement méthodologique suffisamment formalisé pour structurer un pan entier de la pratique ergonomique. Plusieurs modélisations visant à en structurer les différentes étapes sont proposées (voir par exemple Maline, 1994, p. 65 ou encore Daniellou, cité par Barcellini, 2015, p. 187). Barcellini, Van Belleghem et Daniellou (2013) en font une réactualisation contemporaine présentée dans la figure 1.

Figure 1 : Démarche actualisée de l'apport de l'ergonomie en conception'. Figure 1 : Updated approach to the contribution of ergonomics to design

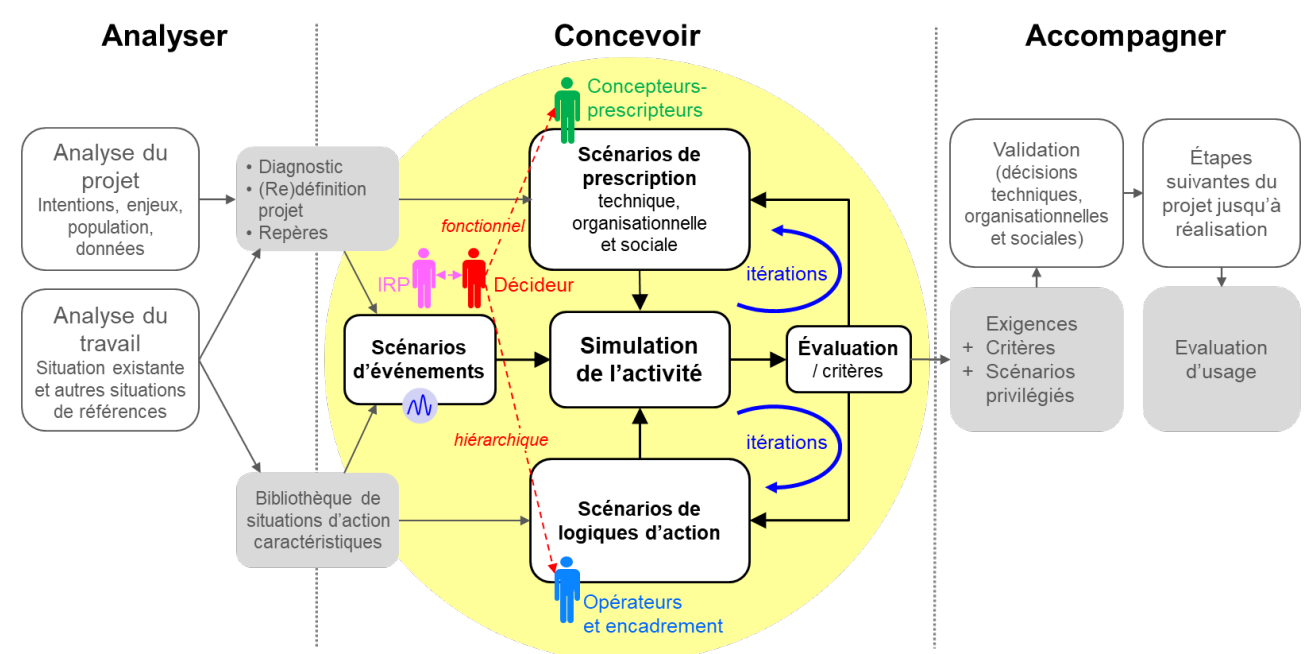

\subsection{Le projet à l'épreuve de l'activité}

La figure 1 représente chronologiquement les trois étapes structurantes d'une intervention ergonomique en conception, depuis l'engagement des premières analyses 
faisant suite à une demande formalisée (et reformulée) jusqu’à la mise en œuvre effective du projet.

6 La première étape combine une analyse du projet (dans ses intentions initiales, ses enjeux économiques et sociaux, l'identification des populations concernées, etc.) à des analyses ergonomiques du travail réalisées sur des situations de référence, couvrant généralement la situation existante, mais aussi des situations voisines, du point de vue des activités mises en œuvre, des situations futures induites par le projet. Cette double analyse doit permettre l'élaboration d'un diagnostic devant orienter le projet grâce l'élaboration de repères pour la conception (Garrigou, Thibault, Jackson, \& Mascia, 2001). Elle doit aussi fournir l'occasion de structurer la démarche participative de projet, au besoin en modifiant ou complétant la structure de projet elle-même (identification des acteurs clé, mise en place d'instances de pilotage et de participation, etc.). Cette étape est aussi, pour l'ergonome, l'occasion de se constituer une bibliothèque de Situations d'Actions Caractéristique (Jeffroy, 1987 ; Garrigou, 1992, Daniellou, 1992 ; Duarte, \& Lima, 2012) qui seront utiles au moment des simulations.

7 La deuxième étape porte explicitement sur la conception participative centrée sur la simulation. Retenons pour l'instant qu'elle consiste à inviter des travailleurs à venir «jouer» leur propre activité dans un contexte prescriptif nouveau, objet de la conception. Ce « jeu», qui est aussi un « jouer » (Hamayon, 2012), soutenu par un support de simulation adapté (une maquette le plus souvent) et l'usage d'un avatar ad hoc, doit permettre d'évaluer les scénarios de prescription proposés par les concepteurs et les modifier le cas échéant, dans l'objectif d'une meilleure adaptation réciproque des choix de prescription à l'activité simulée. À cette occasion, les logiques d'action des travailleurs peuvent elles-mêmes évoluer. Cette double boucle d'itération, portant à la fois sur l'élaboration de scénarios de prescription et le développement de nouvelles logiques d'action des travailleurs, permet de mettre «le projet à l'épreuve de l'activité » là où les modalités classiques de conduites de projet conduisent plus souvent à l'inverse.

8 La dernière étape consiste principalement en la formalisation des résultats de la démarche de conception participative (sous la forme d'un cahier des charges ergonomiques, par exemple) et l'accompagnement du projet dans ses étapes suivantes (qui peuvent intégrer d'autres jalons de conception centrés sur la simulation, par exemple pour poursuivre des études détaillées ou pour anticiper les phases de travaux vers la situation cible (Daniellou, Escouteloup, \& Beaujouan, 2011) jusqu'à sa mise en œuvre effective. Il est pertinent, et de plus en plus accepté par les entreprises, de faire suivre cet accompagnement aux premiers temps de fonctionnement du nouveau système de prescription (dans le cadre d'une évaluation d'usage par exemple), voire en l'inscrivant dans un dispositif de retour d'expérience sur le temps long. Ces dispositifs peuvent aussi contribuer à soutenir des processus d'accompagnement de la conception dans l'usage (Rabardel, 1995). Un champ est d'ailleurs ici ouvert pour y mobiliser des usages innovants de la simulation en « transformation continue ».

9 Ce déroulement méthodologique, conduit dans le cadre d'une intervention inscrite socialement, doit contribuer à une meilleure anticipation des régulations et des possibilités de développement des activités au sein des futures situations. Ce processus de développement est d'ailleurs engagé dès l'étape de conception à travers les simulations réalisées par les travailleurs, permettant leur appropriation anticipée des futures situations de travail. Enfin, l'engagement participatif et contributif de l'ensemble des acteurs (travailleurs, concepteurs, décisionnaires, partenaires sociaux...) contribue à une 
mise en débat du travail renforçant le projet dans ses fondements délibératifs (Barcellini, Van Belleghem, \& Daniellou, 2013).

Mais ces avantages attendus ne sont envisageables que si le processus engagé ne triche pas avec ce qu'il est supposé être, à savoir un processus de conception centré sur la s imulation de l'activité. Or, le terme de simulation est largement employé dans le champ des méthodologies de transformations du travail en général, de la conception en particulier, sans qu'il vise toujours la simulation de l'activité humaine. Ainsi, les ingénieurs développent fréquemment des outils de simulation de process qui, s'ils ont une importance réelle dans les processus de conception, ne peuvent s'apparenter à de la simulation de l'activité (Maline, 1994). Le terme de simulation ergonomique lui-même est ambigu et désigne souvent des formes de simulation de postes ou de situations de travail qui ne mettent pas en œuvre, en tant que telle, une simulation de l'activité humaine (Daniellou, 2007). L'usage d'une maquette non plus, même s'il permet souvent un travail efficace de scénarisation du travail (notamment dans ses aspects prescriptifs), ne suffit pas à constituer à lui seul un dispositif de simulation de l'activité. Enfin, l'irruption des outils de simulation numérique et immersive, ainsi que l'attrait qu'ils peuvent représenter $a$ priori pour les intervenants comme les demandeurs, pose la question de leur pertinence effective comme outils de simulation de l'activité dans un contexte de conception participative: doit-on y voir un élargissement des possibles de la simulation ou s'inquiéter au contraire, sous couvert de nouvelles technologies, de son possible rétrécissement (Arnaud, Fortineau, \& Nahon, 2013)?

11 Ceci incite à préciser ce que désigne, selon nous, la simulation de l'activité. Cette précision ne peut se faire sans un détour par un modèle de l'activité en situation.

\section{L'activité et sa simulation}

\subsection{L'activité en situation}

Travailler, c'est faire face à des événements (Zarifian, 1995). L'activité développée à cette occasion se situe à l'interface entre un système prescriptif et des logiques d'action préélaborées par le sujet, constituant la situation d'activité (voir figure 2). Le système prescriptif est constitué des objectifs à atteindre (la tâche) et de l'ensemble des règles et moyens (techniques, organisationnels et sociaux) mis à disposition du sujet par l'entreprise pour réaliser cette tâche. Il constitue de fait autant un système de contraintes que de ressources pour le sujet agissant. L'activité qu'il développe au sein de ce cadre de prescription, si elle est toujours singulière (en fonction des événements qui, eux-mêmes, le sont), n'en est pas pour autant improvisée. Elle est structurée par des logiques d'action constituées au cours de l'expérience passée du sujet et mobilisables en situation réelle (Van Belleghem, \& Guerry, 2016). L'activité ainsi mise en œuvre produit des effets qui, s'ils sont dirigés principalement vers la réalisation de la tâche, ciblent aussi le sujet, positivement (satisfaction du travail bien fait, reconnaissance, sentiment d'utilité...) ou négativement (fatigue, stress, atteintes à la santé, etc.). Au regard de ces effets constatés, le sujet peut évaluer l'efficacité du couplage entre les caractéristiques du système prescriptif et ses propres logiques d'action et tenter d'agir sur l'un et/ou l'autre pour l'ajuster au mieux. Il existe donc une double boucle de rétroaction de l'activité dirigée d'une part vers ce qui la détermine extrinsèquement, d'autre part vers ce qui la structure intrinsèquement. 
Figure 2 : Modèle de l'activité en situation.

Figure 2 : Activity in situation model

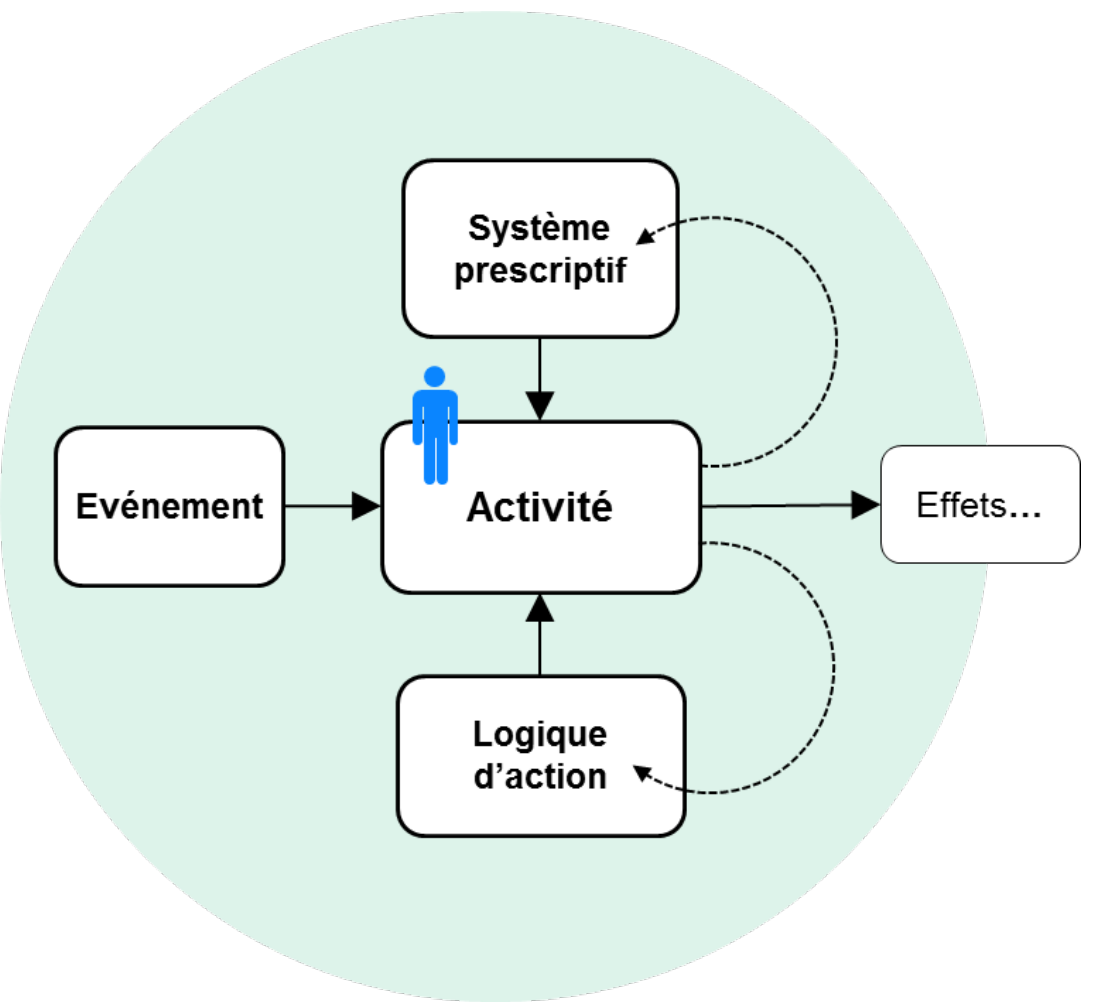

La vraie difficulté pour le sujet agissant dans le contexte d'un travail hétéronome (c'est-àdire déterminé par un tiers dans le cadre d'un lien contractuel de subordination) est d'avoir peu ou pas de possibilités d'agir sur le système de prescription (c'est-à-dire l'ensemble des objectifs, règles et moyens définis par l'entreprise), auquel il est supposé au contraire se conformer strictement. Il ne peut alors compter que sur un ajustement de ses propres logiques d'action qui peuvent trouver leurs limites et s'avérer inadaptées dans un certain nombre de cas, notamment pour faire face à certaines catégories d'événements. C'est à ce moment que peuvent survenir des situations délicates, voire délétères pour le sujet, dans lesquelles les régulations qu'il est amené à réaliser privilégient souvent l'atteinte des objectifs prescrits au détriment des enjeux de santé et de subjectivité qui sont les siens. Ces situations sont significatives d'une inadaptation réciproque du travail et du sujet agissant qu'il s'agit justement d'éviter.

L'intérêt de ce modèle de l'activité en situation est d'offrir une forme à la notion de Situation d'Action Caractéristique (Jeffroy, 1987 ; Garrigou, 1992), centrale en conception ergonomique, mais qui souffre encore d'ambiguïtés dans sa définition et son usage (Thellier, 2013). Définie comme un «ensemble de déterminants dont la présence simultanée va conditionner la structuration de l'activité " (Daniellou, 1992), la Situation d'Action Caractéristique (SAC) permet de décomposer l'activité actuelle, analysée sur des situations de référence voisines de la situation future, en situations élémentaires dont il s'agira d'assurer la transposition dans le projet. Les SAC constituent ainsi les unités élémentaires de l'ergonomie de conception (Maline, 1994).

L'ambiguïté de la notion tient à son caractère composite: en reliant intimement (par définition) ce qui a trait aux déterminants et à l'activité, elle ne facilite pas sa 
transposition dans le projet, où il s'agit justement de pouvoir agir indépendamment sur chacune des composantes de la situation. Le modèle de l'activité en situation présenté cidessus propose de séparer volontairement ces composantes, en distinguant les caractéristiques du cadre prescriptif d'une part, les logiques d'action du sujet d'autre part (qui ne se confondent pas avec l'activité, mais qui en offrent cependant une structuration), le contexte évènementiel enfin (manifestation du réel qui ne s'apparente pas au cadre prescrit). La SAC peut ainsi être comprise comme une instanciation particulière de ces trois composantes à un moment donné (un événement $\mathrm{X}$ dans un contexte $\mathrm{X}$ conduisant à la mobilisation d'une logique d'action $\mathrm{X}$ ) et dont l'activité (sous une forme $\mathrm{X}$ ) rend compte.

L'ergonome peut ainsi identifier des SAC lors de ses observations (SAC actuelles X, Y Z...) et les décomposer en données élémentaires pour anticiper des SAC futures en en faisant varier les différents paramètres sous forme de scénarios (voir figure 3). Ainsi, les événements repérés, mis en lien avec les intentions du projet, permettent d'anticiper les événements futurs qui feront la trame des scénarios d'événements à simuler. Les caractéristiques du système prescriptif actuel, éclairées par les contraintes ou ressources qu'elles offrent à l'activité, orientent les réflexions que les concepteurs pourront ensuite formaliser sous forme de scénarios de prescription. Les logiques de travail repérées, réinvesties dans la situation future, proposent une structuration possible de l'activité tout en s'offrant la liberté d'évoluer pour explorer différents scénarios de logiques d'action. Le dispositif ainsi construit permet de paramétrer des SAC futures selon des configurations volontairement différentes des SAC actuelles (car modifiées par le projet) et d'engager à partir d'elles les simulations de l'activité.

Figure 3 : L'usage des Situations d'Actions Caractéristiques (SAC) dans la simulation. Figure 3 : Use of Characteristic Action Situations (CAS) in simulation

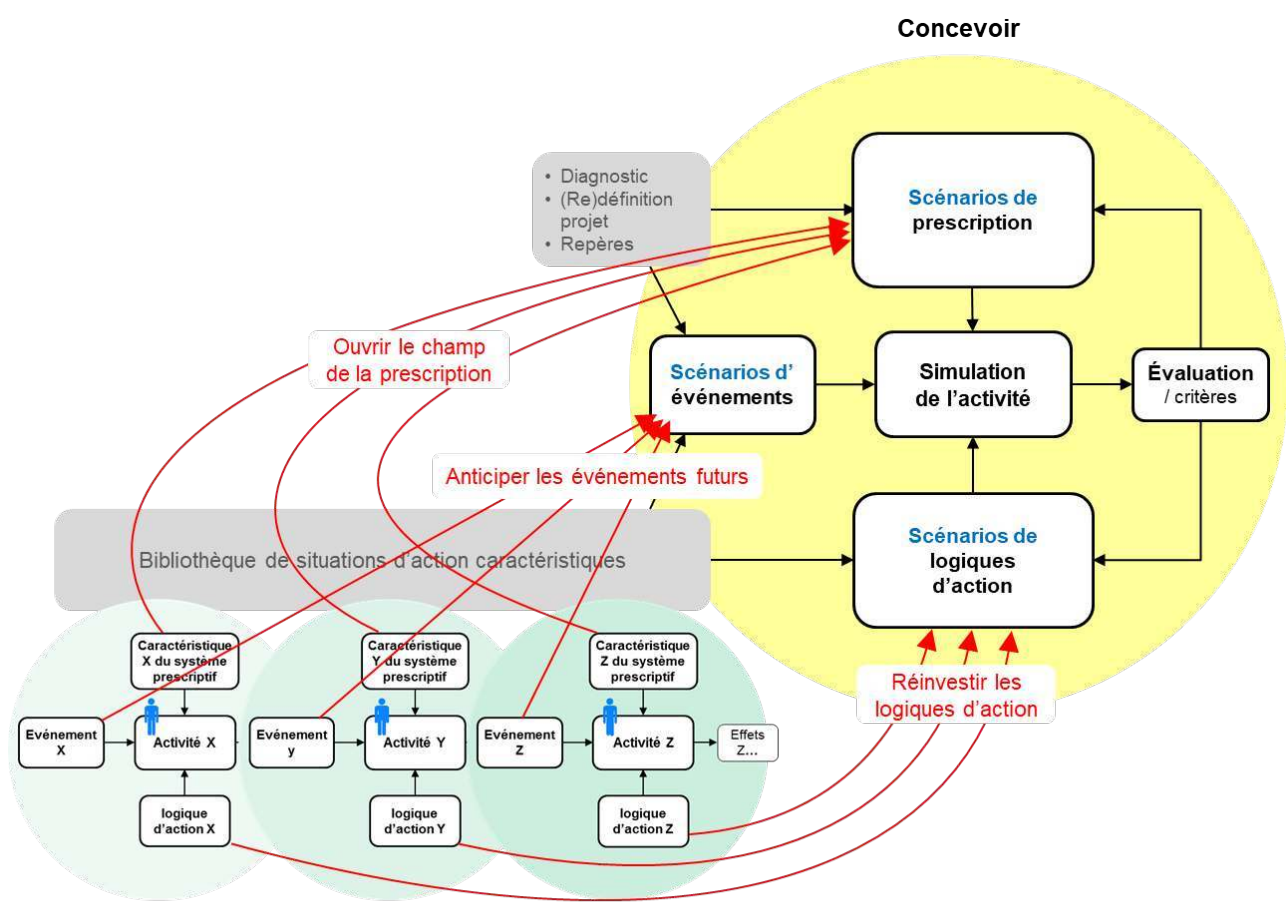


Comme on peut le remarquer, la structure du modèle de la situation de simulation est similaire à celle du modèle de l'activité en situation, à ceci près que les événements, prescriptions et logiques d'action sont présentées sous formes de scénarios, l'activité étant, elle, rendue visible par sa simulation. Cette similitude n'est pas due au hasard. Elle est la condition pour permettre la transposition des situations d'activités actuelles, dans le contexte du projet, en situations simulées. Mais au-delà de cette transposition stricte, il s'agit aussi d'ouvrir le champ des possibles de l'activité en donnant la possibilité aux travailleurs d'agir sur le contexte prescriptif (ce qui leur est généralement refusé dans leur activité quotidienne) tout en faisant évoluer, au besoin, leurs logiques d'action. Autrement dit, il s'agit de remettre en route, et de manière concomitante, les boucles de rétroaction de l'activité sur ses déterminants extrinsèque et intrinsèque. Ces derniers sont dès lors envisagés comme des variables du système (et non plus des constantes) qu'il s'agit d'accorder entre elles pour favoriser la prise en charge des événements à venir dans des conditions acceptables pour l'activité. Sa simulation doit pouvoir en rendre compte. Encore faut-il concevoir et donner forme, concrètement, au dispositif de simulation qui permettra cette exploration des possibles de l'activité.

\subsection{Un dispositif de simulation de l'activité à concevoir et à animer}

Selon sa définition, la simulation vise la reproduction expérimentale ou modélisée d'un phénomène ou d'un système. Dès lors, toute expérience de simulation est supposée reposer sur l'élaboration d'un modèle simulable garantissant une correspondance avec la réalité qu'il est censé représenter. La simulation du travail a cependant cette particularité que la réalité que l'on souhaite se représenter est l'activité. Or, l'activité ne se laisse pas modéliser si facilement, notamment à travers une représentation déterministe (par exemple informatisée) qui serait supposée en prédire les formes rien qu'en modifiant les paramètres d'entrée. Le paradigme épistémologique de l'ergonomie de l'activité nous invite donc à résister à la tentation déterministe et explorer une voie plus constructive de la simulation de l'activité. L'enjeu n'est pas tant, ici, d'imiter un réel possible ou probable que de participer à son élaboration au sein d'une situation de simulation (Béguin, \& WeillFassina, 1997a). Celle-ci engage non pas un modèle, mais des sujets, porteurs de connaissances et d'exigences différentes, dans une activité collective et outillée de simulation. Comme l'exprime Leplat (2000, p. 93), la situation de simulation «implique deux situations: une situation à simuler et une situation qui constitue une simulation de la précédente [...]. À chacune de ces situations est associable une activité ». La situation de simulation peut dès lors être comprise comme une situation d'activité de simulation de l'activité. Van Belleghem (2016), considérant la simulation comme une forme particulière de convocation de l'activité, y repère plus précisément quatre formes d'activités mises en jeu: l'activité cible (celle que l'on cherche à rendre visible), l'activité de simulation (développée par les participants), l'activité simulée (résultat observable de la simulation) et l'activité dialogique, émergeant des interactions entre les participants (ergonome intervenant compris).

Dans l'objectif particulier d'utiliser la simulation en conception, la situation de simulation doit être outillée spécifiquement pour cela. Elle engage des participants aux profils volontairement différenciés (opérateurs et opératrices, concepteurs, décideurs, représentants du personnel...), s'appuie sur un support de simulation conçu selon des exigences spécifiques et met en œuvre une méthodologie d'animation en capacité 
d'amorcer et de maintenir la mise en mouvement des boucles de rétroaction de l'activité sur ses déterminants en itérant scénarisation et simulation. L'ergonome intervenant prend donc une part significative dans l'animation de la simulation, en invitant les travailleurs à "jouer» leur activité en suivant les scénarios d'événements qu'il a préélaborés, en s'assurant à tout moment de la vraisemblance des situations produites, en garantissant la continuité spatiale, temporelle et cognitive de l'activité simulée, en faisant évaluer collectivement le caractère acceptable (ou non) de l'activité simulée devant conduire à valider ou réviser le scénario de prescription. Il a vocation aussi, en collaboration avec les concepteurs et décideurs, à animer et orienter le travail de conception, c'est-à-dire la scénarisation des éléments de prescription, en alternance avec les phases de simulation. La conception d'un support adapté à ce double objectif de scénarisation et de simulation est ici primordiale.

Le support de simulation est un objet intermédiaire particulier (Broberg, Andersen, \& Sem, 2011) qui peut prendre plusieurs formes. Une reproduction pleine échelle du système en cours de conception permet la simulation d'une activité en "grandeur réelle », permettant aux opérateurs d'éprouver dans leur corps la future situation (Daniellou, 2007). Ce procédé est pertinent pour concevoir des postes de travail matériellement et géographiquement circonscrit où les dimensions physiques du travail sont prédominantes. Il ne peut se faire, cependant, que si le projet est suffisamment avancé pour avoir d'ores et déjà déterminé les caractéristiques structurantes du poste et en faire ce type de prototypage. Une reproduction à échelle réduite (maquette) est plus adaptée pour élaborer des projets de grande taille (atelier, bâtiment, process industriel...) et permet d'élaborer des scénarios même dans les phases amont du projet. Enfin, certains types de projets nécessiteront des formes de maquettages plus abstraits et sans notion d'échelle, par exemple pour des projets à dominante immatérielle (organisation, management...) ou pour simuler des dimensions non physiques du travail (activités cognitives, communications, traitements de dossiers, relations de coopération, etc.). On notera l'intérêt qu'il y a, grâce à des supports abstraits ou à échelle réduite, à positionner les participants dans une vision allo-centrée du projet plutôt qu'égocentrée, telle que privilégiée par les simulateurs pleine échelle. Cette vision allo-centrée ("God's view ») place ainsi chacun des participants, et particulièrement les opérateurs-trices, dans une position dominante vis-à-vis du projet qui n'est plus seulement celle de l'utilisateur, voire de l'exécutant, mais bien celui de concepteur, en capacité d'agir sur lui et d'en maitriser, au moins en partie, le devenir².

21 Quel que soit le type de support utilisé, sa conception doit répondre à trois exigences fondamentales (Van Belleghem, 2012) devant permettre la représentation et l'évolution des éléments de prescription sous forme de scénarios, la modification collective des scénarios de prescription et la simulation de l'activité. L'exigence de simulation doit être particulièrement traitée pour dépasser le stade de la simulation langagière consistant à inviter les travailleurs à décrire verbalement leur activité (Béguin, 2004). Il est en effet difficile, pour un sujet, de mettre en mots son activité. Cela l'est d'autant plus lorsqu'il s'agit de verbaliser une activité qui n'existe pas encore. La seule vue d'une maquette représentant le futur contexte prescriptif ne suffit pas, en l'occurrence, à lever cette difficulté, voire peut la tromper en réduisant par trop la complexité du travail. Dès lors, le risque d'une faible projection dans la nouvelle situation est important. Il conduit fréquemment le sujet à verbaliser les tâches qu'il devra réaliser plutôt que son activité en déroulement. L'usage du pronom indéfini neutre «on » (« on traite », « on distribue », « on 
vérifie », etc.) doit, de ce point de vue, servir de marqueur d'alerte pour l'animateur. Il traduit en effet le plus souvent une description du process plutôt que de l'activité. Si cette description peut avoir un certain intérêt (le process est un objet de conception qu'il faut savoir définir), elle écarte l'activité de simulation de son objectif qu'est la simulation de l'activité. L'activité de simulation de l'activité doit donc être précisée dans ses mécanismes ${ }^{3}$ pour être mieux outillée.

\subsection{L'activité de simulation de l'activité}

Simuler sa propre activité, voire celle des autres, est somme toute assez naturel chez l'être humain (Le Guyader, 2012). C'est même une de ses particularités que d'interagir avec son environnement en élaborant des hypothèses sur son action et ses effets possibles avant même de l'avoir engagée, et dont il pourra évaluer par comparaison l'effectivité après coup, voire au cours même du déroulement de l'action pour mieux s'y ajuster (Berthoz, 1997). Ces mécanismes, de nature biologique et sélectionnés au cours de l'évolution, s'appuient sur une mise en lien complexe et subtile du corps (squelette, muscles, sens...) et du cerveau, permettant à ce dernier d'anticiper les conséquences prévisibles de l'action engagée en matière de retours sensoriels. Toute action, tout geste, est d'abord simulée dans sa dynamique par le cerveau, même lorsque cette action est entravée physiquement. L'expérience joue ici un rôle primordial dans la façon dont elle permet au sujet d'emmagasiner des séquences d'action aux effets prédictibles et de les restituer en situation tout en les adaptant aux évolutions de l'environnement. Autrement dit, «le cerveau n'est pas une machine réactive, c'est une machine proactive qui projette sur le monde ses interrogations» (ibid., p. 7). C'est aussi un simulateur d'action qui utilise la mémoire pour prédire le futur.

La difficulté consistant à reproduire ce mécanisme en situation de simulation tient à ce qu'il se réalise principalement hors de la conscience. En effet, le cerveau ne traite que les écarts éventuels entre le mouvement simulé et sa réalisation effective, mais pas les similitudes (Davezies, 2012). Cette régulation dite en feed-forward, qui ne traite que ce qui ne se passe pas comme prévu, permet un ajustement de l'action particulièrement efficace et économe. Mais cette efficience a son revers: elle opacifie l'action pour le sujet luimême, à la conscience de qui elle ne remonte pas. C'est ce qui explique que la mise en mots de l'activité, fut-elle particulièrement réglée, et même pour des actions a priori très simples, soit particulièrement compliquée. Le sujet agissant ne se sentant pas agir, il lui est difficile de parler de son action ${ }^{4}$. Mais il peut la montrer.

La situation de simulation doit pour cela être conçue comme un lieu de mise en œuvre d'une action préalable à sa mise en mots et non l'inverse. Ce n'est pas le langage, et avant lui la pensée, qui permet de simuler l'action, mais bien la dynamique de l'action qui offre au langage un point d'appui auquel s'accrocher. Et si «évoquer l'action, c'est la simuler intérieurement ", (ibid., p. 8), nous faisons l'hypothèse que ce processus de simulation intérieure peut être "extériorisé ", au sein d'un dispositif adapté, pour mieux se rendre visible et intelligible par tous. Fournir un avatar au sujet invité à simuler son activité est, de ce point de vue, primordial (Van Belleghem, 2012). Un avatar est une figurine (de type Playmobil@ ou Lego@) ou un objet spécifiquement choisi qui, saisi et manipulé par le sujet, lui permettra d'incarner son activité sur le support de simulation représentant, lui, le contexte dans lequel "agit» cet avatar. Cet artifice matériel permet de distinguer clairement ce qui tient de l'activité du sujet (une activité de manipulation de l'avatar) de 
ce qui tient de «l'activité » de l'avatar, que l'ensemble des participants peut observer en train "d'agir ", aidé en cela par les verbalisations du salarié venant soutenir son activité simulée. Il s'agit bien là d'une activité (du sujet) de simulation de l'activité (de l'avatar). Mais au-delà de cet " artifice ", il y a un processus psychologique profond à l'œuvre dans cette manipulation. Ce processus dit de cognition découplée (Le Guyader, 2012) s'appuie sur une capacité de l'être humain à "produire des inférences à partir de prémisses non réelles " (p.108), c'est-à-dire à se représenter des situations en tous points cohérentes avec la réalité mais dans laquelle certains éléments sont volontairement occultés ou rajoutés sans que ce changement modifie le système d'inférences habituel. C'est par ce principe que les enfants peuvent «jouer à faire semblant ", s'attribuer des pouvoirs magiques ou transformer n'importe quel objet en petite voiture qu'ils vont pouvoir piloter à l'envi. Et c'est par ce principe que des scénarios imaginés, fussent-ils tout à fait incongrus, peuvent être explorés par l'activité de celui qui se prête à ce jeu de la simulation. C'est cette capacité, dans l'évolution humaine, qui aurait permis à des chasseurs-cueilleurs de " planifier une expédition de chasse, réfléchir à la fabrication d'outils, procéder à des échanges... » ( ibid., p. 108). Autrement dit, à concevoir leurs situations d'activités à venir.

\subsection{Du « je » au « on » et retour}

La saisie de l'avatar s'accompagne d'un autre effet remarquable: l'usage du pronom personnel « je » suivi d'un verbe d'action conjugué au présent de l'indicatif, parfois au futur: " je commence par regarder mes mails... ", "je m'informe des consignes du jour...", «je traite le dossier... ». L'action simulée est ainsi accompagnée de sa verbalisation. Le langage, ici, décrit et précise l'action en cours, mais ne la précède pas. La simulation est d'abord l'engagement d'une action, via l'avatar, qui permet sa mise en mots et facilite son intelligibilité, tant pour le sujet que pour les participants. Et l'on sait que «la compréhension des verbes d'action active, dans les aires prémotrices et motrices, les zones correspondant au mouvement évoqué » (Davezies, 2012, p. 8). La simulation d'une action active ainsi les aires cérébrales de cette même action, tant pour le sujet lui-même que pour ceux qui l'observent et l'écoutent. Simuler n'est donc pas feindre : c'est agir pour de vrai.

On notera ici l'usage du «je» comme un marqueur pertinent d'une simulation de l'activité qui ne soit pas qu'une simulation de la tâche caractérisée, comme cela a été vu précédemment, par l'usage du « on ». Le passage de l'un à l'autre, cependant peut être vu comme une itération intéressante, et même attendue, entre le travail de simulation de l'activité et celui de scénarisation des choix de prescription. À l'activité simulée " je vais prendre les consignes... », on peut répondre : «où prend-t-on les consignes? ». Au «je » de l'activité renvoie donc un « on » de la tâche. Ce re-questionnement invite à une réflexion collective devant aboutir à un choix de conception qui enrichira le scénario de prescription du projet: "on prend les consignes ici!». Dès que ce choix sera acté, la simulation pourra se poursuivre, redonnant une place au « je » de l'activité simulée : « dès que j'ai pris mes consignes, je me dirige vers mon poste... ». La boucle de rétroaction de l'activité sur ses déterminants est ici à l'œuvre (voir figure 4), observable notamment par le passage $\mathrm{du}$ « je » au « on » avant de revenir au « je », etc. C'est bien le projet, ici, qui est mis à l'épreuve de l'activité. Et c'est bien l'activité qui structure le projet, en lui donnant une pertinence objectivable par le travail réel. 
On constatera que le « on », qui appelle ici une logique de prescription (« où prend-t-on les consignes?»), renvoie par ailleurs à la structuration des futures logiques d'action des sujets, c'est-à-dire à un "on » discutable, objet d'un débat collectif, sur lequel peut se construire le genre professionnel (Clot, 2006) des sujets («nous, on propose de s'y prendre comme ça»). C'est ici la boucle de rétroaction du côté des logiques d'action que la simulation de l'activité permet d'engager, structurant les pratiques professionnelles des acteurs du travail. C'est-à-dire leur métier.

Figure 4 : le « je » et le « on » comme marqueurs de l'activité simulée et des boucles de rétroaction à l'œuvre.

Figure 4 : "I" and "we" as markers of simulated activity and feedback loops at work

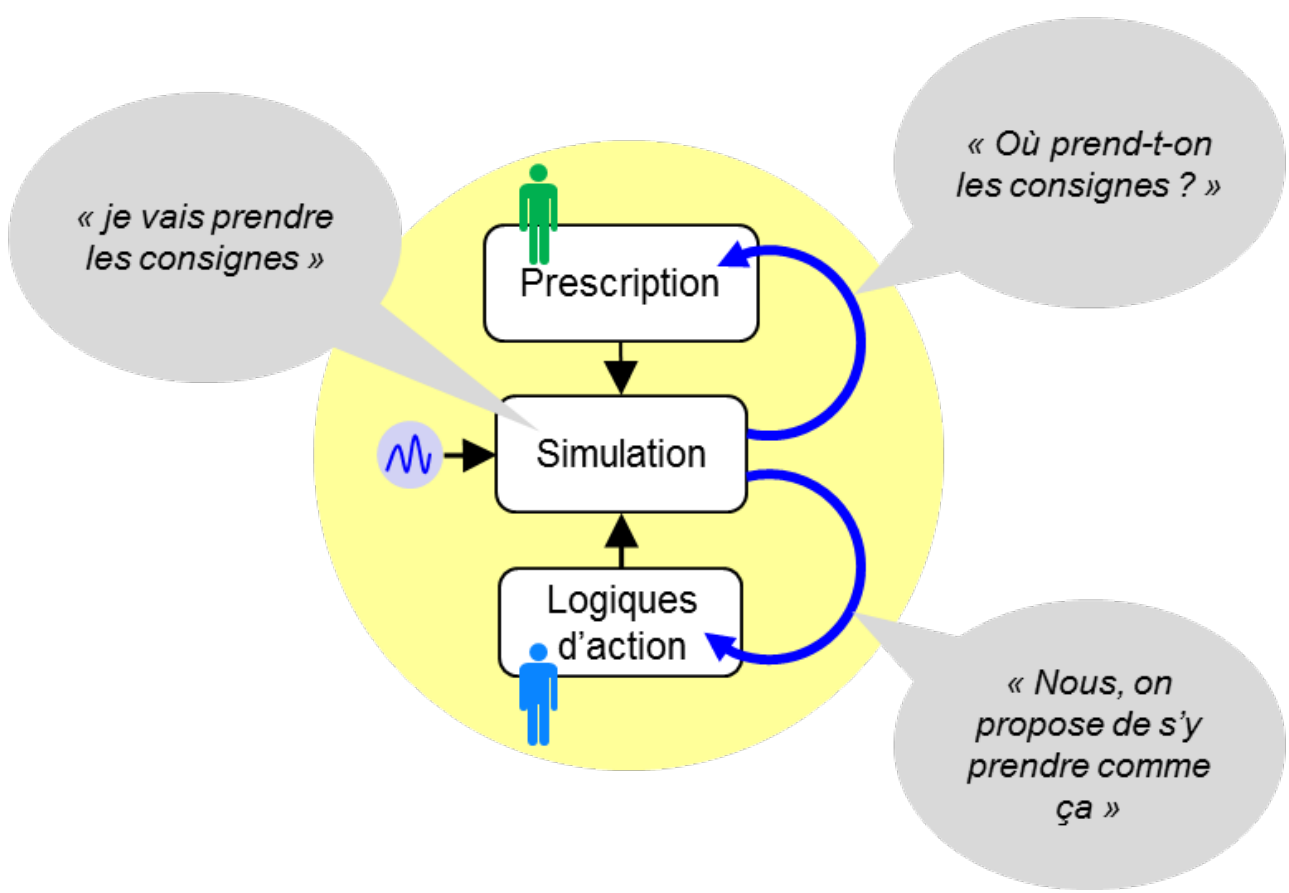

Les itérations successives entre le « je » et le " on » lors de la simulation visent ainsi, en les construisant concomitamment, la convergence du système de prescription et du métier, intimement reliés par l'activité. Dans le champ du travail, les perspectives ouvertes par ce mécanisme sont potentiellement vastes. Il faut les dresser.

\section{Les perspectives de la simulation}

L'ouverture des usages de la simulation peut être envisagée selon deux grandes orientations. La première porte sur l'exploitation des potentialités offertes par les boucles de rétroaction de l'activité sur ses déterminants. La seconde sur la diversification des objets de conception qu'une approche centrée sur l'activité autorise.

\subsection{Exploitation des facultés de rétroaction de l'activité sur ses déterminants}

La faculté de la simulation à « mettre en route » les boucles de rétroaction de l'activité sur ses déterminants offre différentes applications possibles pour transformer le travail. 
Trois perspectives d'application peuvent être plus particulièrement exploitées selon qu'elles mettent en œuvre l'une et/ou l'autre des boucles de rétroaction (voir figure 5).

Figure 5 : Exploitation différenciée des boucles de rétroaction.

Figure 5 : Differentiated exploitation of feedback loops

(a) Correction

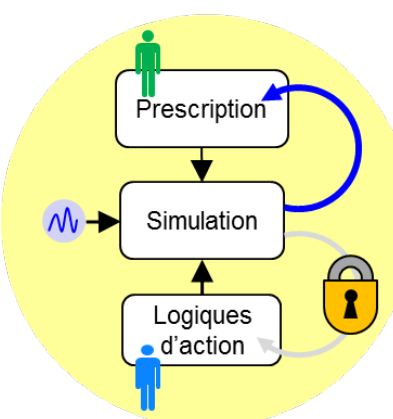

(b) Conception

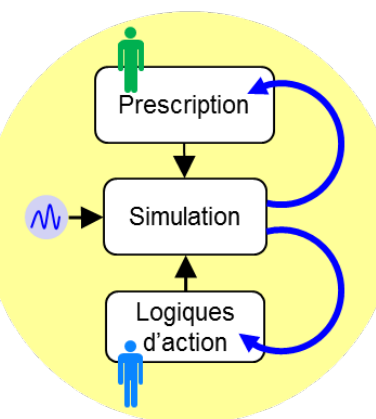

(c) Appropriation

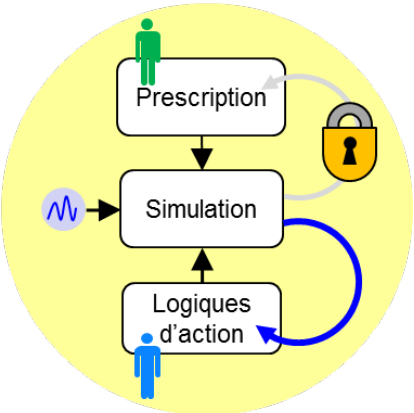

La première perspective (voir figure $5 \mathrm{a}$ ) vise la correction des situations de travail. Elle cherche à adapter le système de prescription à des logiques d'action identifiées comme acceptables mais insuffisamment servies par leurs conditions de mises en œuvre. Dès lors, l'enjeu de la simulation consiste à rétroagir sur le système de prescription sans modifier les logiques d'action des sujets. On mesure cependant la difficulté, voire l'incongruité, qui réside dans cette façon de procéder. Les logiques d'action des sujets sont en effet intimement liées au système de prescription avec lequel ils interagissent, y compris lorsque ce dernier, prenant peu en compte les contraintes du travail réel, les oriente vers des formes coûteuses ou délétères. Il y a peu de chances que des logiques d'action acceptables aient réellement pu émerger dans un tel système, sauf pour tenter d'en surmonter les contraintes. Dès lors, il n'y a pas lieu de les reconduire, mais bien plutôt de les transformer. Ce point peut expliquer la tombée en désuétude de l'ambition ergonomique liée à la correction des situations de travail, pour lui préférer leur conception.

La perspective liée à la conception (voir figure 5b) s'appuie sur la mise en œuvre simultanée des deux boucles de rétroaction. Il s'agit d'agir en même temps sur le système de prescription et sur le champ des logiques d'actions des sujets, en les scénarisant l'un par rapport à l'autre. L'enjeu consiste à explorer le champ des possibles de l'un et l'autre sans s'interdire a priori de voies particulières. Il y a donc ici un enjeu d'innovation rendu possible par la créativité de l'agir (Joas, 2008) propre au travail quotidien, mais trop souvent entravé par les contraintes du système prescriptif imposé. La simulation de l'activité offre ici l'occasion d'une mise en œuvre de cette créativité de l'agir dans un contexte où les paramètres de la situation sont suffisamment souples pour lui permettre de s'exprimer pleinement et d'explorer les voies de l'agir qui lui semblent opportunes. Une évaluation du caractère acceptable de l'activité ainsi simulée est réalisée par les participants et relance les recherches de meilleures solutions dans différentes directions, contribuant à leur diversification. Les solutions les plus convaincantes sont sélectionnées et affinées jusqu'à correspondre au meilleur compromis entre les exigences des participants. On assiste ici à une forme d'innovation darwinienne (Pick, 2011) par la mise en œuvre d'un processus accéléré de diversification / sélection portant sur l'activité ellemême. Cet usage simultané des deux boucles de rétroaction dans une perspective 
d'émulation de l'innovation dans le travail (Durand, Moaty, \& Tiffon, 2014) se prête donc particulièrement à l'enjeu de conception.

Une troisième perspective, que nous proposons de nommer appropriation (voir figure 5c) peut être envisagée en gelant les possibilités de rétroaction sur le système de prescription pour explorer exclusivement le champ des possibles du côté des logiques d'action. C'est fréquemment le cas, dans un projet, lorsque le passage d'un jalon fige certains choix de conception, réduisant ainsi ses marges de manœuvre, mais augmentant d'autant sa connaissance (Midler, 2004), permettant ainsi son appropriation par les sujets. C'est le cas aussi lorsqu'il s'agit d'accueillir un système déjà conçu (une technologie, un robot, une application...) dans un écosystème existant et qui devra l'intégrer sans le rejeter. La perspective est ici pleinement développementale (Falzon, 2013) et vise la construction d'une expérience partagée apte à s'approprier et «mettre à sa main » ce nouvel élément de la situation de travail. Cette expérience peut prendre plusieurs formes et a intérêt à faire l'objet d'une exploration large pour scénariser différentes logiques d'action possibles avant d'en sélectionner les plus pertinentes, qui structureront le cœur de l'appropriation. Cette perspective peut bien sûr être envisagée dans un objectif explicite de formation, visant à orienter l'élaboration des logiques d'action vers des scénarios prédéfinis (et non plus à en explorer le champ des possibles) et constituant des compétences spécifiques à acquérir. Cette perspective couvre les simulations à visée didactique, qu'elles s'appuient sur des simulateurs classiques (de type simulateur de conduite full scale privilégiant la reproduction à l'identique de la situation réelle) ou qu'elles cherchent, à travers des simulateurs de résolution de problèmes, à reproduire un problème significatif présent dans le travail et en capacité de générer des apprentissages ciblés chez des sujets invités à s'y confronter (Pastré, 2005). Cette perspective est particulièrement utile, dans un projet, pour étendre l'apprentissage du système conçu à l'ensemble des travailleurs concernés, au-delà des seuls travailleurs ayant eu à participer aux groupes de travail visant sa conception. Un accompagnement ergonomique d'une conduite de projet sur l'ensemble de son déroulement aura donc intérêt à anticiper le passage de l'usage de la simulation en conception à celui de l'appropriation, y compris formative.

L'exploitation des potentialités offertes par les boucles de rétroaction de l'activité doit par ailleurs pouvoir s'associer à une extension des objets de la conception.

\subsection{Extension du domaine de la conception}

La conception porte classiquement sur des artefacts: outils, postes, lieux de travail, bâtiments... Cette orientation détermine mécaniquement le profil des concepteurs sollicités : designer, ingénieurs, architectes, bureaux d'études... C'est à cette occasion que la référence à l'activité comme élément centralisateur du travail peut être mise en second plan, voire occultée complétement, dans la conduite de projet. Engager, avec les demandeurs, une réflexion sur une conception centrée sur l'activité, c'est donner l'occasion de penser les artefacts moins à travers leur matérialité ou leur symbolisme qu'à travers l'activité qui les met en œuvre, les justifie et les modèle ${ }^{5}$. C'est aussi, possiblement, décentrer l'objet de la conception. Le travail dans toute sa complexité et dans toutes ses dimensions peut ainsi devenir un objet de conception. Certaines de ces dimensions peuvent apparaître comme particulièrement structurantes et se voir accorder un statut particulier dans le projet, voire en constituer l'objet à part entière. 
L'organisation, le management, la discussion sur le travail, la subsidiarité, le métier, la confiance, l'autonomie, la gouvernance... peuvent ainsi devenir des objets de conception légitimes. Et dont la mise en œuvre peut être simulée.

La simulation organisationnelle (Van Belleghem, 2012 ; Daniellou, Legal, \& Promé, 2014, Mellon, 2015) vise ainsi à accompagner les projets de conception des organisations du travail. L'organisation est entendue ici, dans la lignée de la théorie de la régulation sociale de Reynaud (1988) comme un système de règles combinant des règles formelles (ou règles de contrôle) définies extérieurement pour être respectées par le groupe auxquelles elles s'appliquent, et des règles informelles (ou règles de métier) élaborées par et pour le groupe d'exécution selon sa propre stratégie (de Terssac, 1992). Cette approche reconnait qu'à l'organisation du travail comme système de règles prescrites lui est toujours opposé un travail d'organisation, qui prend sa source dans l'activité et les interactions sociales entre acteurs. Ce processus, produisant des règles effectives (et donc observables), permet de faire face à la diversité des situations jamais entièrement prévisibles par le système de prescription à lui seul. Concevoir une organisation, c'est donc élaborer les conditions de fonctionnement de ce double système de règles, portant autant sur les règles prescrites (en tant qu'artefacts à concevoir) que les règles de métier (en tant que logiques d'action à développer). La simulation s'y prête donc particulièrement, dont l'objectif peut être défini ainsi : élaborer des règles formelles favorisant le développement de règles effectives acceptables pour l'activité. Encore faut-il qu'elle dispose d'un dispositif propre à soutenir cette dynamique, et notamment d'un support de simulation adapté. Le support (ou le système de supports) devra ainsi être en capacité de représenter le système de règles prescrites en cours de conception (ex: organigramme fonctionnel, process, logigramme de tâches, tableau de planification, etc.), d'autoriser la modification collective de ces règles par les participants au cours de l'avancement des échanges, et de permettre la simulation de l'activité des travailleurs grâce à l'usage d'un avatar adapté. Le choix de ce dernier devra faire l'objet d'un soin particulier pour savoir incarner les dimensions de l'activité concernées par le projet d'organisation.

La simulation managériale (Van Belleghem, \& Guerry, 2016 ; Besnard, \& Levy, 2017) vise plus précisément à (re)concevoir les situations de travail des managers. Traditionnellement, les évolutions du management sont envisagées sous l'angle d'une évolution des managers eux-mêmes, individuellement (par exemple par des méthodes formatives : formation au leadership, à la communication...) ou collectivement (par exemple par des méthodes de team-building: sorties sportives, escape-games...). Ces approches sont sous-tendues par l'idée que les managers «font leur poste » par euxmêmes quand, dans la réalité des entreprises, ces postes sont largement prescrits en termes de périmètre d'action, de dimensionnement des équipes, de délégations de pouvoirs, d'objectifs à atteindre, d'indicateurs à remonter, etc. Ces déterminants du travail des managers constituent l'organisation du management. Il y a dès lors un enjeu à envisager l'évolution du management à travers l'évolution de son organisation (et non des managers eux-mêmes), qui peut être ainsi reconnue comme un objet de conception à part entière. L'ergonome peut ainsi engager une analyse approfondie des activités des managers, en identifier les logiques d'action et les déterminants structurels, et proposer de transformer l'organisation du management dans le cadre d'une conduite de projet centrée sur la simulation de l'activité des managers et visant à agir sur les règles d'organisation du management. Cette approche offre notamment l'opportunité d'une élaboration collective et structurée des logiques d'action des managers là où, comme on 
l'a vu, ces derniers sont le plus souvent livrés à eux-mêmes dans cette exigence de développement. L'itération simultanée des deux boucles d'itération, l'une sur les règles d'organisation du management, l'autre sur les logiques d'action des managers, entrainée par la simulation de leur activité, constitue ici un principe de convergence des déterminants intrinsèque et extrinsèque $d u$ management rarement possible dans l'activité quotidienne des managers.

La simulation gouvernantielle est une autre variante de la simulation rendant compte de la diversification en cours de ses usages possibles. Proposition émergente dans le champ du conseil (et qui gagnerait à être approfondie dans le champ de la recherche), elle vise à désigner le système de gouvernance des entreprises comme un objet de conception qu'une intégration de l'activité des décideurs permettrait de mieux instruire. À l'instar des managers, l'activité des décideurs est déterminée par un ensemble de règles (de fonctionnement de l'entreprise, de ses instances décisionnelles et gestionnaires - Conseil d'Administration, Codir, Comex, de ses instances sociales, etc.). De fait, les décideurs n'ont qu'un pouvoir limité dans la mesure où ils sont eux-mêmes engagés dans le système de gouvernance qu'ils ont contribué à développer. L'enjeu stratégique d'un système de gouvernance réside le plus souvent dans l'autonomie qu'il sait octroyer aux différents acteurs de l'entreprise pour contribuer au processus décisionnel sans entamer pour autant la légitimité des dirigeants. Entre l'entreprise bureaucratique (strictement topdown) et l'entreprise libérée (idéalement bottom-up), il existe un large panel de systèmes décisionnels possibles, recourant de façon plus ou moins volontariste au principe de subsidiarité (devant permettre la prise de décision au niveau pertinent le plus bas de l'organisation), à la séparation des pouvoirs ou à la participation des salariés. Nous faisons l'hypothèse que ces systèmes pourraient être mieux instruits en s'autorisant à scénariser plus systématiquement les formes qu'ils pourraient prendre et en simulant l'activité décisionnelle des collaborateurs qui y seraient engagés. Des expérimentations de cette approche ont commencé, notamment dans le champ universitaire, dont nous espérons qu'elles pourront produire des résultats suffisamment structurants pour faire l'objet de publications futures.

La mise en place d'espaces de discussion sur le travail a récemment fait l'objet d'un intérêt renouvelé (Detchessahar, 2011) et généré des demandes multiples et diversifiées de la part des entreprises, administrations et collectivités. Ces espaces de discussion, s'ils sont entendus comme des espaces de régulation du travail, peuvent contribuer à structurer le travail d'organisation au quotidien (Detchessahar, 2013) et participer à l'affirmation du principe de subsidiarité dans les organisations contemporaines (Rocha, Mollo, \& Daniellou, 2017). Encore s'agit-il que la discussion porte bien sur le travail réel et contribue à sa transformation. Disposer d'un outillage méthodologique permettant de convoquer l'activité (par des méthodes d'entretien d'explicitation ou d'auto-confrontation par exemple) est ainsi un enjeu central de ces espaces de discussion (Van Belleghem, 2016). La simulation de l'activité est une des méthodes possibles, portant cependant le débat moins sur le travail actuel que sur le travail futur possible. Casse et Caroly (2017) la mettent œuvre pour soutenir un « retour d'expérience effectif » au sein de collectifs de travail intervenant sur des situations potentiellement à risques dans des tunnels routiers. Le dispositif conçu, dit de « simulation croisée " par leurs concepteurs, permet à plusieurs collectifs d'acteurs de simuler leur activité collective face à un même scénario d'événement proposé (un feu de véhicule en bord de route par exemple). Les résultats des simulations sont ensuite mis en commun et les écarts entre les propositions émises par 
les groupes sont discutés collectivement. Ce processus, en organisant la confrontation sur une diversité de situations événementielles dans un temps limité, permet d'accélérer l'élaboration de règles communes à travers la construction d'une expérience partagée. La simulation ne contribue donc pas seulement à l'anticipation de l'activité future, mais participe tout autant à la construction de l'expérience des sujets, dans une perspective développementale.

Au-delà des exemples présentés ci-dessus, de nombreuses autres dimensions du travail peuvent être identifiées que la simulation de l'activité doit pouvoir contribuer à mieux instruire. La sagacité des ergonomes doit continuer à les identifier et leur créativité à inventer les dispositifs propres à en faire des « objets de conception » légitimes et utiles à l'amélioration du travail.

\section{Conclusion}

41 Malgré le recul lié à trente ans d'usage de la simulation en conception ergonomique, la méthodologie conserve des potentialités inexplorées. Les évolutions du travail contemporain invitent instamment à les révéler. Industrie du futur, digitalisation, économie dite collaborative (ou plateformisation), entreprise libérée... transforment les structures profondes du travail. Le besoin d'anticiper les formes que ces mutations induisent sur l'activité humaine est primordial. Mais encore plus sûrement se pose l'exigence d'orienter ces transformations au regard de ce dont l'activité est porteuse, y compris dans ses formes encore insoupçonnées. Il y a bien, ici, un enjeu à mobiliser une forme de créativité de l'agir sur laquelle appuyer les innovations du travail, et non de soumettre l'activité à des innovations technologiques et/ou sociétales pensées en dehors d'elle. C'est à cet enjeu que la simulation de l'activité a vocation à répondre.

Dans cet objectif, trois axes de recherche sur la simulation de l'activité seraient à envisager. Le premier doit porter sur l'extension de ses champs d'application: gouvernance, décision stratégique, dialogue social, démocratie dans le travail... doivent pouvoir être reconnus comme des objets de conception légitimes qu'une approche centrée sur l'activité peut aider à désigner comme tels. Le deuxième vise la consolidation des démarches d'accompagnement du changement que ces nouveaux objets de conception appellent. Le troisième vise la formalisation du processus de créativité de l'agir en jeu dans la simulation. Gageons que l'intérêt renouvelé dont fait preuve la simulation de l'activité aujourd'hui saura nourrir ces axes de recherche.

\section{BIBLIOGRAPHIE}

Andersen, S.N., \& Broberg, O. (2015). Participatory ergonomics simulation of hospital work systems : The influence of simulation media on simulation outcome. Applied Ergonomics, 51, 331-342. DOI : 10.1016/j.apergo.2015.06.003 
Andersen, S.N., \& Broberg, O. (2016). A framework of knowledge processes in participatory simulation of hospital work systems. Ergonomics, 60(4), 1-39. DOI : 10.1080/00140139.2016.1212999

Arnaud, S., Fortineau, E., \& Nahon, S. (2013). Simulations immersives en réalité virtuelle : une innovation technologique au service de l'intervention ergonomique ? Communication présentée au $48^{\text {ème }}$ Congrès de la Société d'Ergonomie de Langue Française, Paris, France. https://ergonomieself.org/wp-content/uploads/2017/02/SELF-Recueil-Actes-2013.pdf

Barcellini, F. (2015). Développer des interventions capacitantes en conduite du changement : comprendre le travail collectif de conception, agir sur la conception collective du travail. Habilitation à Diriger des Recherches, Université de Bordeaux.

Barcellini, F., Van Belleghem, L., \& Daniellou, F. (2013). Les projets de conception comme opportunité de développement des activités. In P. Falzon (Ed.) Ergonomie constructive. pp. 191-206. Paris : PUF.

Béguin (2004). L'ergonome, acteur de la conception. In P. Falzon (Ed.) Ergonomie. pp. 375-390. Paris : PUF.

Béguin, P., \& Weill-Fassina, A. (Eds.) (1997a). La simulation en ergonomie : Connaître, agir et interagir. Toulouse : Octarès.

Béguin, P., \& Weill-Fassina, A. (1997b). De la simulation des situations de travail à la situation de simulation. In P. Béguin \& A. Weill-Fassina (Eds.) La simulation en ergonomie : Connaître, agir et interagir. pp. 5-28. Toulouse : Octarès.

Besnard, L., \& Levy, E. (2017). Comment outiller une démarche de réflexion sur la gestion temporelle par la simulation managériale ? Communication présentée au $52^{\text {ème }}$ Congrès de la Société d'Ergonomie de Langue Française, Toulouse, France.

Berthoz, A. (1997). Le sens du mouvement. Paris : Odile Jacob.

Braatz, D., \& Paravizo, E. (2017). Articulating intermediary objects in the design process to enable ergonomics intervention. Communication présentée au 12th International Symposium on Human Factors in Organizational Design and Management (ODAM), Banf, Canada.

Braatz, D., Tonin, L., Fontes, A., \& Menegon, N. (2013). Digital human modeling and simulation for ergonomics workspace design : two Brazilian cases. Communication présentée au $2^{\text {nd }}$ International Digital Human Modeling Symposium, Ann Harbor, Michigan, U.S.A.

Broberg, O., Andersen, V., \& Seim, R. (2011). Participatory ergonomics in design processes : the role of boundary objects. Applied Ergonomics, 42(3), 464-472. DOI : 10.1016/j.apergo.2010.09.006

Cahour, B., Salembier, P., \& Zouinar, M. (2016). Analyzing lived experience of activity. Le Travail Humain, 79(3), 259-284. DOI : 10.3917/th.793.0259

Casse, C., \& Caroly, S. (2017). Enrichir le retour d'expérience par les espaces de débat : l'exemple d'une recherche-intervention chez un exploitant de tunnels routiers. Le Travail Humain, 80(3), 259-284. DOI : 10.3917/th.803.0259

Clot, Y. (2006). La fonction psychologique du travail. Paris : PUF.

Clot, Y., Faïta, D., Fernandez, G., \& Scheller, L. (2000). Entretiens en auto-confrontation croisée : une méthode en clinique de l'activité. Perspectives interdisciplinaires sur le travail et la santé, 2(1), $1-8$. DOI : $10.4000 /$ pistes.3833

Daniellou, F. (1992). Le statut de la pratique et des connaissances dans l'intervention ergonomique de conception. Thèse d'habilitation à diriger des recherches. Éditions du LESC, Bordeaux. 
Daniellou, F. (2004). L'ergonomie dans la conduite de projets de conception de systèmes de travail. In P. Falzon (Ed.), Ergonomie. pp. 359-373. Paris : PUF.

Daniellou, F. (2007). Des fonctions de la simulation des situations de travail en ergonomie. Activités, 4(2), 77-83. DOI : 10.4000/activites.1696

Daniellou, F., Escouteloup, J., \& Beaujouan, J. (2011) Phasage des travaux et organisations transitoires : quels rôles pour l'ergonome? Activités, 8(1), 26-43. DOI : 10.4000/activites.2503

Daniellou, F., Legal, S., \& Promé, M. (2014). Organisational simulation : anticipating the ability of an organisation to cope with daily operations and incidents. In O. Broberg, N. Fallentin, P. Hasle, P.L. Jensen, A. Kabel, M.E. Larsen \& T.Weller (Eds) Communication présentée au 11th International Symposium on Human Factors in Organizational Design and Management (ODAM). Copenhagen : Danmarks Tekniske Universitet.

Davezies, P. (2012). Enjeux, difficultés et modalités de l'expression sur le travail : point de vue de la clinique médicale du travail. Pistes, 14(2), 1-18. DOI : 10.4000/pistes.2566

Falzon, P. (2013). Ergonomie Constructive. Paris : PUF.

Detchessahar, M. (2011). Santé au travail. Quand le management n'est pas le problème, mais la solution... Revue française de gestion, 2011(5), 89-105.

Detchessahar, M. (2013). Faire face aux risques psychosociaux : quelques éléments d'un management par la discussion. Négociations, 2013(1), 57-80. DOI : 10.3917/neg019.0057

Duarte, F., \& Lima, F. (2012). Anticiper l'activité par les configurations d'usage : proposition méthodologique pour conduite de projet. Activités, 9(2), 22-47, DOI : 10.4000/activites.314 Durand, J.-P., Moatty, F., \& Tiffon, G. (Eds.) (2014). L'innovation dans le travail. Toulouse : Octarès. Garrigou, A. (1992). Les apports des confrontations d'orientations socio-cognitives au sein de processus de conception participatifs. Thèse de doctorat en ergonomie, Paris : Cnam.

Garrigou, A., Thibault, J.-F., Jackson, M., \& Mascia, F. (2001). Contributions et démarche de l'ergonomie dans les processus de conception. Pistes, 3(2), 1-20. DOI : 10.4000/pistes.3725 Hamayon, R. (2012). Jouer. Une étude anthropologique. Paris : La Découverte.

Hauret, D., Donnot, J., \& Van Belleghem, L. (2016). Intégration de la simulation de l'activité sur support tangible dans le processus de conception de l'interface homme-système du Rafale. Communication présentée à la $15^{\text {ème }}$ Conférence Ergo'IA, Biarritz, France.

Heddad, N. (2016). L'espace de l'activité, de l'analyse à la conception. Thèse de doctorat en ergonomie, Paris : Cnam.

Jeffroy, F. (1987). Maîtrise de l'utilisation d'un système micro-informatique par des utilisateurs non informaticiens. Thèse de doctorat en ergonomie, Paris : Cnam.

Joas, H. (2008). La créativité de l'agir. Paris : Editions du Cerf.

Le Guyader, H. (2012). Penser l'évolution. Paris : Imprimerie Nationale Editions.

Leplat, J. (2000). L'analyse psychologique de l'activité en ergonomie. Paris : Presses Universitaires de France.

Maline, J. (1994). Simuler le travail, une aide à la conduite de projet. Lyon : ANACT.

Mellon, N. (2015). Simulation organisationnelle : matérialiser la dématérialisation. Communication présentée au $50^{\text {ème }}$ congrès de la Société d'Ergonomie de Langue Française, Paris, France. 
Midler, C. (2004). L'auto qui n'existait pas. Management des projets et transformation de l'entreprise. Paris : Dunod.

Mollo, V., \& Falzon, P. (2004). Auto and allo-confrontation as tools for reflective activities. Applied Ergonomics, 35 (6), 531-540. DOI : 10.1016/j.apergo.2004.06.003

Pastré, P. (2005). Apprendre par la résolution de problèmes : le rôle de la simulation. Dans P. Pastré (dir.) Apprendre par la simulation. De l'analyse du travail aux apprentissages professionnels. Pp .18-40. Toulouse : Octarès.

Pick, P. (2011). Un paléoanthropologue dans l'entreprise. S'adapter et innover pour survivre. Paris : Eyrolles.

Rabardel, P. (1995). Les hommes et les technologies, approche cognitive des instruments contemporains. Paris : Armand Colin.

Raspaud, A. (2014). De la compréhension collective de l'activité réelle à la conception participative de l'organisation : plaidoyer pour une intervention ergonomique capacitante. Thèse de doctorat en ergonomie, Paris : Cnam.

Reynaud J.-D. (1988). Les régulations dans les organisations : régulation de contrôle et régulation autonome. Revue française de Sociologie, 29(1), 5-18.

Rocha, R., Mollo, V., \& Daniellou, F. (2017) Le débat sur le travail fondé sur la subsidiarité : un outil pour développer un environnement capacitant. Activités, 14(2), 1-26. DOI : 10.4000/ activites.2999

Terssac, G. (de) (1992). Autonomie dans le travail. Paris : PUF.

Thellier, S. (2013). Les situations d'actions caractéristiques. Mémoire d'Analyse Bibliographique, ERG255. Paris : Cnam.

Theureau, J., \& Pinsky, P. (1984). Paradoxe de l'ergonomie de conception et logiciel informatique. Revue des Conditions de Travail, 9, 25-31.

Van Belleghem, L. (2012). Simulation organisationnelle : innovation ergonomique pour innovation sociale. In M.F. Dessaigne, V. Pueyo, et P. Béguin (Eds.) Innovation et travail. Sens et valeurs du changement. Actes du 47ème congrès de la Société d'Ergonomie de Langue Française du 5, 6 et 7 septembre 2012. Lyon : Editions du Gerra.

Van Belleghem, L. (2016). Eliciting activity : a method of analysis at the service of discussion. Le Travail Humain, 79(3), 285-306. DOI : 10.3917/th.793.0285

Van Belleghem, L., \& Guerry, M.-H. (2016). De la simulation organisationnelle à la simulation managériale. Communication présentée au 51ème Congrès de la Société d'Ergonomie de Langue Française, Marseille, France.

Vermersch, P. (1994). L'entretien d'explicitation. Issy-Les-Moulineaux : ESF.

Zarifian, P. (1995). Le travail et l'événement, essai sociologique sur le travail industriel à l'époque actuelle. Paris : L'Harmattan.

\section{NOTES}

1. Ce schéma, publié initialement dans Van Belleghem (2012) et Barcellini, Van Belleghem, \& Daniellou (2013) a fait l'objet d'une adaptation dans Van Belleghem, \& Guerry (2016) ayant conduit à distinguer clairement les scénarios d'événements des scénarios de logiques d'action, 
regroupés à l'origine sous l'appellation commune scénarios d'action. Le contexte événementiel, les caractéristiques du système de prescription et les formes prises par les logiques d'action peuvent être ainsi définies indépendamment les uns des autres.

2. Hauret, Donnot, \& Van Belleghem (2016) décrivent ainsi un support de simulation pour la conception des interfaces du futur cockpit de l'avion de chasse Rafale qui a savamment évité l'usage du simulateur utilisé traditionnellement en formation (et dans lequel les pilotes doivent se glisser) pour lui préférer une représentation du théâtre d'opérations dans son ensemble (frontières, zones de combat, corridors aériens, camps et avions ennemis...) intégrant une maquette à échelle réduite du Rafale qu'un pilote est invité à manipuler manuellement. La simulation consiste alors à déplacer l'avion dans le champ de bataille selon les stratégies du pilote et à lui faire verbaliser, à chaque minute de la mission simulée, les besoins en informations que le cockpit doit renvoyer en fonction de la situation vue ici dans sa globalité et ses enjeux tactiques (vision allo-centrée), et non depuis le seul siège de l'avion (vision égocentrée).

3. C'est-à-dire les principes et conditions spécifiques de mise en œuvre, dans le fonctionnement humain, d'une activité de simulation (se projeter dans la situation, manipuler un avatar, verbaliser, choisir ses mots, chercher à se mettre dans la peau de son personnage...) permettant la mise à jour d'une activité simulée (travailler «effectivement " dans la situation projetée, accomplir des tâches, gérer des aléas, faire face à des événements...). Cette distinction entre activité de simulation et activité simulée reprend celle faite par Van Belleghem (2016) entre activité de convocation et activité convoquée.

4. De nombreux travaux en ergonomie et psychologie ont contribué à définir les bases théoriques et méthodologiques permettant l'accès à la conscience pré-réfléchie par la verbalisation (Vermersch, 1994, Cahour, Salembier, \& Zouinar, 2016) ou des méthodologies spécifiques de type auto-confrontation (Clot, Faïta, Fernandez, \& Scheller, 2000, Mollo, \& Falzon, 2004). Cependant, ces méthodologies cherchent davantage à accéder aux ressorts de l'activité vécue plutôt que de l'activité future.

5. Heddad (2015) montre ainsi avec une grande justesse comment l'espace de travail peut être compris moins comme un lieu défini matériellement qu'une émergence de l'activité elle-même, le recomposant à son gré, et justifiant de le renommer « l'espace de l'activité ».

\section{RÉSUMÉS}

Le recours à la simulation de l'activité en conception fait l'objet d'un intérêt renouvelé tant en France qu'à l'international, notamment au Brésil et au Danemark. Cet article se propose, en s'appuyant sur les principes acquis de la simulation de l'activité en ergonomie, d'en approfondir les mécanismes pour mieux donner à voir son potentiel d'usage et en ouvrir ses perspectives d'application. Il est organisé en trois parties. La première rappelle les acquis fondamentaux de la simulation depuis trente ans jusqu'à ses formalisations et développements actuels. La deuxième tente d'approfondir théoriquement les mécanismes de la simulation de l'activité en lien avec l'activité elle-même. La troisième explore les perspectives actuelles de la simulation, en activant d'abord dans différentes directions ses facultés de rétroaction sur le système de prescription et sur les logiques d'action des sujets agissants, en élargissant ensuite les objets de conception qu'elle permet d'approcher. 
There is renewed interest in the use of activity simulation in design, not only in France but internationally, particularly in Brazil and Denmark. This article examines the principles established through activity simulation in ergonomics, with a view to developing the mechanisms underlying activity simulation in order to shed more light on its potential use, and to open up perspectives for its application. It is organized into three parts. The first offers a reminder of the fundamental achievements of simulation over the last thirty years, up to its current formalization and development. The second seeks to provide a more in-depth theoretical analysis of activity simulation mechanisms as they relate to the activity itself. The third explores current perspectives for simulation, first by activating its capacities in different directions for feedback on the prescription system and on the action logics of active subjects, and then by broadening the objects of conception it allows us to approach.

INDEX

Mots-clés : ergonomie, conception, simulation de l'activité, simulation organisationnelle

Keywords : ergonomics, design, activity simulation, organizational simulation

\section{AUTEUR}

\section{LAURENT VAN BELLEGHEM}

Professeur associé CNAM-CRTD-Ergonomie.

Ergonome consultant, REALWORK, 5, rue Jules Vallès, 75011 Paris

laurent.vanbelleghem@realwork.fr 